\title{
Méthode de mesure de l'efficacité des procédés de nettoyage et de désinfection des surfaces ouvertes
}

\author{
A Brouillaud-Delattre 1, A Kobilinsky 2, O Cerf 3* \\ Avec la collaboration technique de $S$ Aligé ${ }^{3}$, G Gerlot ${ }^{3}$ et JM Herry ${ }^{3}$ \\ ${ }^{1}$ Association pour le développement de la recherche dans l'industrie laitière, \\ 34, rue de Saint-Pétersbourg, F-75382 Paris Cedex 08; \\ 2 Laboratoire de biométrie, INRA, route de Saint-Cyr, F-78026 Versailles Cedex; \\ ${ }^{3}$ Laboratoire de génie de l'hygiène et des procédés alimentaires, INRA, \\ 25, avenue de la République, F-91300 Massy, France
}

(Reçu le 10 juin 1993; accepté le 16 septembre 1993)

\begin{abstract}
Résumé - L'article décrit une méthode de mesure de l'efficacité du nettoyage et de la désinfection des surfaces ouvertes, c'est-à-dire non nettoyables par écoulement de liquide en circuit fermé. Elle utilise un robot pour assurer la reproductibilité des opérations. Elle emploie des souillures laitières représentatives des conditions industrielles, et des micro-organismes traceurs pour quantifier séparément le nettoyage (spores de bactéries thermophiles) et la désinfection (culture mixte de bactéries végétatives). Les traceurs de désinfection forment un biofilm. Un plan d'expérience multifactoriel est utilisé pour mettre en évidence l'influence et les interactions des facteurs expérimentaux tels que la durée d'application, la concentration de produit de nettoyage et de désinfection, la nature et la quantité de souillure, la nature et l'état de la surface, etc. Une expérimentation avec un produit détergentdésinfectant combiné permet de valider la méthode.
\end{abstract}

hygiène / nettoyage / désinfection / surface / mesure / efficacité

Summary - Method for measuring the efficiency of cleaning and disinfection processes on open surfaces. This paper describes a method for measuring the efficiency of cleaning and disinfection processes on open surfaces (which cannot be cleaned in situ by fluid flowing in a closed circuit). A robot was used for ensuring reproducibility of operations. Representative dairy soils (rennet curd of $45 \%$ fat ultrafiltration retentate, saint-paulin cheese solubilized in $2 \%$ trisodium citrate) were spread over a plate including 16 samples of the studied surface material. The latter was AISI $304 \mathrm{~L}$ stainless steel, with a roughness $R_{a}$ of 0.25 or $0.73 \mu \mathrm{m}$. Microbial tracers were added to the soils for separate quantification of cleaning (thermophilic bacterial spores of Bacillus thermophilus var calidolactis) and disinfection (mixed culture of 2 mesophilic vegetative bacteria: Lactococcus lactis subsp lactis, Leuconostoc mesenteroides). Disinfection tracers formed a biofilm in $18 \mathrm{~h}$ at $20^{\circ} \mathrm{C}$. A multifac-

* Correspondance et tirés à part 
torial experimental design with 6 blocks including 2 repetitions was used. It enabled to show the influence and interactions of experimental factors such as application time, product concentration, soil type and weight, surface type and roughness, etc. The method was validated with a combined detergent-disinfectant. The method can be used in dairy and non-dairy contexts, since it can be applied to any representative soils and microorganisms (whether or not they form a biofilm), to any detergents and/or disinfectants, and with any experimental design.

\section{hygiene / cleaning / disinfection / surface / measurement / efficiency}

\section{INTRODUCTION}

Il existe une méthode admise pour mesurer l'efficacité des procédés de nettoyage dans les circuits fermés nettoyables en place (Holah et al, 1992). Elle utilise une boucle expérimentale permettant de mesurer l'aptitude au nettoyage des pièces d'équipements. Cette boucle, qui existe à de nombreux exemplaires en Europe (dont au moins 3 en France), pourrait être utilisée pour mesurer l'efficacité de procédés de désinfection. Pour ces derniers par ailleurs, existent des normes, notamment pour décrire la mesure de l'efficacité des désinfectants appliqués à des bactéries mélangées à une souillure séchée sur une surface (Afnor, 1989). Cependant, on peut se demander si une souillure séchée est représentative des conditions industrielles où règne une forte humidité relative, ou bien où l'on trouve des films d'eau sur les surfaces. En effet, dans de telles conditions, des biofilms se forment, c'est-à-dire des communautés microbiennes adhérant à une surface au sein d'une couche muqueuse. Or, on sait que les bactéries des biofilms sont plus résistantes aux désinfectants que les bactéries en suspension (Costerton et al, 1978; Characklis et Marshall, 1990 ; Cerf et Carpentier, 1992). Mais on ignore si la résistance des bactéries séchées est représentative de celle des bactéries des biofilms.

Pour les surfaces ouvertes, c'est-à-dire les surfaces extérieures des équipements, et les sols, murs et plafonds, il n'existe aucune méthode disponible ni aucune norme à notre connaissance. Le présent article décrit une méthode destinée à remplir cette lacune, et ayant le cahier des charges suivant :

- elle utilise une souillure choisie pour être représentative de conditions industrielles données, transformée en biofilm par la croissance d'une population bactérienne mixte ;

- elle utilise un robot pour assurer la reproductibilité de l'étalement de la souillure, et de l'application du procédé de nettoyage et de désinfection ;

- elle permet d'étudier des procédés de nettoyage ou de désinfection, ou dans le cas des procédés combinés, de distinguer l'efficacité du nettoyage et celle de la désinfection ;

- elle permet l'emploi d'un plan d'expérience multifactoriel, afin de mettre en évidence l'influence de chaque facteur expérimental et d'éventuelles interactions entre facteurs (Goupy, 1988).

\section{MATÉRIEL ET MÉTHODES}

\section{Surface}

On utilise de l'acier inoxydable $304 \mathrm{~L}$ avec 2 finis de surface, de rugosité moyenne Ra égale à $0,25 \pm 0,03 \mu \mathrm{m}$ ou $0,73 \pm 0,05 \mu \mathrm{m}$ (rugosimètre Diavite DT 100). Pour les essais, 16 
éprouvettes circulaires de diamètre $50 \mathrm{~mm}$ sont disposées dans une plaque du même matériau, de façon à ce que leur surface et celle de la plaque soient de niveau.

\section{Micro-organismes}

\section{Marqueur du nettoyage}

Des spores de Bacillus stearothermophilus var calidolactis C 953 (CNRZ 41) recommandées comme marqueur de la quantité de souillures (Galesloot et al, 1967) sont préparées selon la norme AFNOR T72-230 (AFNOR, 1989) modifiée pour adapter à cette espèce thermophile les températures de sporulation $\left(58^{\circ} \mathrm{C}\right)$ et de pasteurisation $\left(90^{\circ} \mathrm{C}\right)$. Avant emploi, les spores sont activées à $110^{\circ} \mathrm{C}$ pendant $5 \mathrm{~min}$.

\section{Marqueur de l'efficacité globale}

Deux souches de bactéries lactiques sont utilisées: Lactococcus lactis subsp lactis CNRZ 189 et Leuconostoc mesenteroides NCDO 523. Les bactéries sont préparées par 2 repiquages en bouillon de Elliker et al (1956) à $30^{\circ} \mathrm{C}$ (pour $L$ lactis), et de de Man, Rogosa et Sharpe (1960) (pour $L$ mesenteroides) à $22^{\circ} \mathrm{C}$, pendant $24 \mathrm{~h}$.

\section{Souillures}

Deux souillures sont mises au point. Leur composition leur assure une bonne homogénéité et une facilité d'étalement sur la surface étudiée :

- I'une est à base de caillé frais à $45 \%(\mathrm{~m} / \mathrm{m})$ de matière grasse (MG) obtenu par coagulation présure d'un rétentat gras obtenu par ultrafiltration; on y ajoute $5 \%(\mathrm{v} / \mathrm{v})$ de suspension de marqueur de nettoyage, et $2,5 \%(\mathrm{v} / \mathrm{v})$ de chaque marqueur de désinfection, en suspension dans du citrate trisodique à $2 \%(\mathrm{~m} / \mathrm{v})$ (BrouillaudDelattre, 1993).

- l'autre est à base de fromage de saint-paulin à $45 \%(\mathrm{~m} / \mathrm{m}) \mathrm{MG}$, solubilisé dans du citrate trisodique à $2 \%(\mathrm{~m} / \mathrm{v})$; on y ajoute $10 \%(\mathrm{v} / \mathrm{v})$ de marqueur de nettoyage, et $7,5 \%(\mathrm{v} / \mathrm{v})$ de chaque marqueur de désinfection en suspension dans du citrate trisodique à $2 \%(\mathrm{~m} / \mathrm{v})$ (BrouillaudDelattre, 1993).

Après mélange, la souillure est appliquée sur la surface, et incubée $18 \mathrm{~h}$ dans une étuve climatique $\left(20^{\circ} \mathrm{C}, 90 \%\right.$ d'humidité relative).

\section{Robot}

Un robot programmable à 3 axes linéaires, piloté par ordinateur (Charlyrobot, Annecy) est utilisé pour encrasser ou appliquer le produit de nettoyage et désinfection, à l'aide d'outils appropriés. Les figures 1 et 2 présentent respectivement une vue générale du robot et de son support, et la surface avec les 16 éprouvettes.

\section{Procédé de nettoyage et désinfection}

Un produit commercial, alcalin chloré, appliqué sous forme de mousse, a été utilisé. À la concentration de $1 \%(\mathrm{v} / \mathrm{v})$, le $\mathrm{pH}$ du produit est égal à 11,75 et la concentration en chlore est égale à $400 \mathrm{mg} / \mathrm{l}$. À la concentration de $3 \%$ $(v / v)$, le $\mathrm{pH}$ du produit est égal à 12,25 et la concentration en chlore est égale à $1,2 \mathrm{~g} / \mathrm{l}$. Le procédé comprend l'application de la mousse ci-

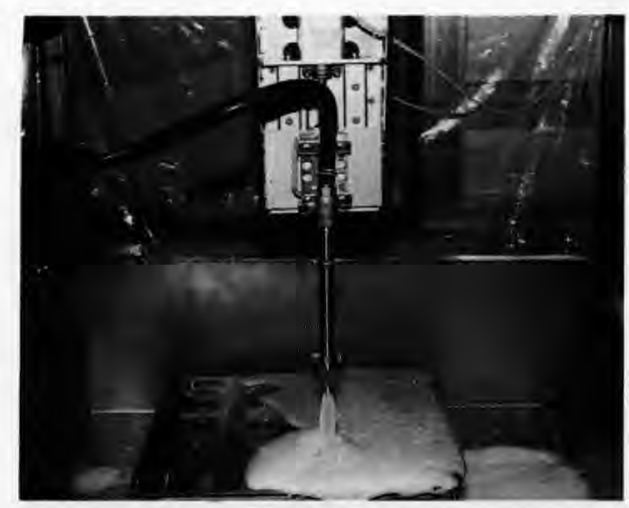

Fig 1. Vue du robot: le canon à mousse (fixé à l'axe vertical) se déplace au-dessus de la plaque comportant les éprouvettes.

View of the robot: foam spout is moved over the samples by means of vertical and horizontal movement of the fixture. 
dessus et le rinçage par de l'eau de ville $(0,26$ $\pm 0,2 \mathrm{l} / \mathrm{s}$ ), jusqu'à disparition visuelle de la mousse. Ces opérations sont effectuées à température ambiante $\left(16-18^{\circ} \mathrm{C}\right)$.

\section{Dénombrement des micro-organismes adhérant aux surfaces}

Les dénombrements des micro-organismes adhérant aux éprouvettes témoins et aux éprouvettes traitées se font après détachement des micro-organismes par insonification, suivie d'une homogénéisation. L'insonification est réalisée de façon aseptique, les éprouvettes étant placées face souillée vers le bas, dans $20 \mathrm{ml}$ d'une solution stérilisée de citrate trisodique à $2 \%(\mathrm{~m} / \mathrm{v})$ contenant $0,2 \%(\mathrm{~m} / \mathrm{v})$ de polysorbate 80 , à $\mathrm{pH}=8,1$. On utilise des bechers de 250 $\mathrm{ml}$ de forme haute, à raison de 4 à la fois. On emploie un appareil Ultrasonic $250 \mathrm{Ney}$, de capacité $5,7 \mathrm{I}$, de fréquence $40 \mathrm{kHz}$, réglé à la puissance maximale (non indiquée), contenant $1,5 \mathrm{I}$ de solution de polysorbate 80 à $0,3 \%(\mathrm{~m} / \mathrm{v})$ préalablement dégazée par insonification pendant $3 \mathrm{~min}$. L'insonification dure $25 \mathrm{~min}$. La température initiale est de $15^{\circ} \mathrm{C}$ et la température finale est $<30^{\circ} \mathrm{C}$. Après insonification, la solution est transférée aseptiquement dans des sacs en polyéthylène et soumise à un broyage péristaltique de $90 \mathrm{~s}$ dans un appareil Stomacher (Prolabo).

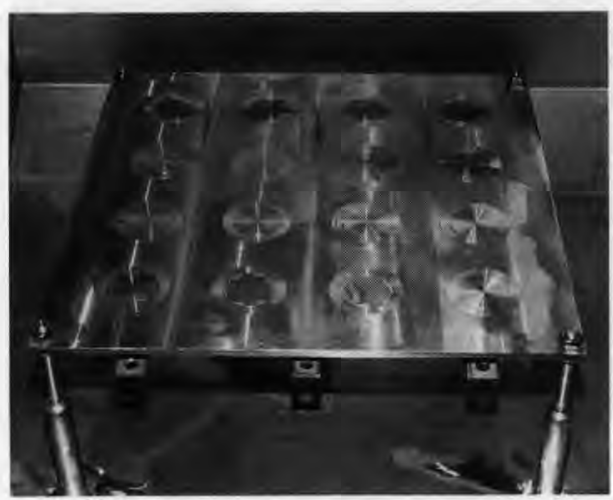

Fig 2. Disposition des 16 éprouvettes. Set-up of the 16 samples.

\section{Plan d'expérience}

Le plan d'expérience est un plan multifactoriel complet, à 5 facteurs, et 2 niveaux par facteurs. Les expériences sont regroupées en blocs, un bloc correspondant à l'application d'un même procédé sur les 16 éprouvettes d'une même plaque. Le plan comporte 6 blocs notés de $A$ à $F$, dont 2 ( $B$ et $C$ ) sont des répétitions. L'emploi de blocs consiste à regrouper les expériences de telle manière que les effets principaux (et certaines interactions) ne soient pas faussés par des variations d'ensemble. Les essais correspondant aux répétitions permettent de vérifier qu'il n'y a pas de variation des réponses entre les blocs. Les facteurs étudiés sont :

- le type de souillure $\left(S_{0}=\right.$ caillé, $S_{1}=$ saintpaulin):

- la quantité de souillure déposée sur les éprouvettes $\left(Q_{0}=0,01\right.$ g/éprouvette, $Q_{1}=0,10$ g/éprouvette) :

- le temps d'action du produit $\left(T_{0}=15 \mathrm{~min}, T_{1}=\right.$ $30 \mathrm{~min})$;

- la concentration de produit $\left(\mathrm{C}_{0}=1 \%(\mathrm{v} / \mathrm{v})\right.$, $\mathrm{C}_{1}=3 \%(\mathrm{v} / \mathrm{v})$;

- la rugosité des éprouvettes $\left(R_{0}=0,25 \mu \mathrm{m}\right.$, $\left.R_{1}=0,73 \mu \mathrm{m}\right)$.

\section{Présentation des résultats}

\section{Efficacité de nettoyage et de désinfection}

La masse de souillure après application du procédé $M_{\mathrm{f}}$ est estimée à partir de $M_{\mathrm{i}}$, masse initiale de souillure mesurée par pesée, de $N_{i}$, quantité initiale de spores dans la souillure dénombrée sur les éprouvettes témoins, et de $N_{f}$, nombre final de spores, par l'équation suivante:

$$
M_{\mathrm{f}}=M_{\mathrm{i}} \cdot N_{\mathrm{f}} / N_{\mathrm{i}}
$$

L'efficacité du nettoyage $E_{n}$ est exprimée en nombre de réductions décimales (divisions par 10) de la masse de souillure :

$$
E_{n}=\log _{10}\left(M_{i} / M_{f}\right)
$$


L'efficacité globale du procédé $E_{g}$ est estimée à partir du nombre initial ni et du nombre final $n_{f}$ d'unités formant colonies (UFC), par l'équation:

$$
E_{g}=\log _{10}\left(n_{i} / n_{f}\right)
$$

L'efficacité de la désinfection $E_{d}$ est calculée par l'équation :

$$
E_{d}=E_{g}-E_{n}
$$

\section{Influence des facteurs expérimentaux}

L'influence des facteurs expérimentaux est présentée d'une part par leur caractère significatif à $5 \%\left({ }^{*}\right)$, hautement significatif à $1 \%\left(^{* \star}\right)$ ou très hautement significatif à $0,1 \%\left({ }^{* * *}\right)$; d'autre part, par leur pourcentage d'explication de la variance (défini comme le degré de contribution d'un effet ou d'une interaction sur la totalité des effets et des interactions étudiés).

\section{RÉSULTATS}

\section{Détachement de la souillure, et dénombrements microbiologiques avant application du procédé}

Des essais préliminaires sont faits avec 4 éprouvettes à 6 reprises. Les résultats montrent que la récupération de la

Tableau I. Efficacité du traitement avec une mousse détergente et désinfectante : chaque case présente l'efficacité globale, et, entre parenthèses, l'efficacité du nettoyage et l'efficacité de la désinfection.

Efficiency of a combined detergent-disinfectant applied as a foam. Global efficiency and, in brackets,

\begin{tabular}{|c|c|c|c|c|c|c|}
\hline Souillure & $\begin{array}{l}\text { Concen- } \\
\text { tration }\end{array}$ & $\begin{array}{l}\text { Temps } \\
\text { (min) }\end{array}$ & $R_{0}$ & $R_{1}$ & $R_{0}$ & $R_{1}$ \\
\hline \multirow[t]{2}{*}{ Caillé } & $1 \%$ & $\begin{array}{l}15 \\
30 \\
30\end{array}$ & $\begin{array}{l}2,4(1,3+1,1) \\
2,3(0,8+1,5) \\
1,4(0,5+0,9)\end{array}$ & $\begin{array}{l}1,4(0,5+0,9) \\
1,2(0,5+0,7) \\
1,1(0,6+0,5)\end{array}$ & $\begin{array}{l}1,1(0,4+0,7) \\
0,4(0,25+0,15) \\
0,3(0,15+0,15)\end{array}$ & $\begin{array}{l}0,3(0,1+0,2) \\
0,3(0,2+0,1) \\
0,4(0,2+0,2)\end{array}$ \\
\hline & $3 \%$ & $\begin{array}{l}15 \\
15 \\
30\end{array}$ & $\begin{array}{l}1,5(0,75+0,75) \\
1,1(0,35+0,75) \\
1,1(0,5+0,6)\end{array}$ & $\begin{array}{l}1,8(1,0+0,8) \\
1,7(0,9+0,8) \\
1,2(0,6+0,6)\end{array}$ & $\begin{array}{l}0,5(0,3+0,2) \\
0,6(0,4+0,2) \\
0,4(-0,3+0,7)\end{array}$ & $\begin{array}{l}0,8(0,7+0,1) \\
0,8(0,6+0,2) \\
1,0(-0,1+1,1)\end{array}$ \\
\hline \multirow[t]{2}{*}{$\begin{array}{l}\text { Saint- } \\
\text { paulin }\end{array}$} & $1 \%$ & $\begin{array}{l}15 \\
30 \\
30\end{array}$ & $\begin{array}{l}0,6(0,4+0,2) \\
2,0(0,7+1,3) \\
3,5(1,8+1,7)\end{array}$ & $\begin{array}{l}0,4(-0,2+0,6) \\
2,9(1,5+1,4) \\
2,7(1,5+1,2)\end{array}$ & $\begin{array}{l}0,4(0,3+0,1) \\
3,3(1,3+2,0) \\
2,8(1,0+1,8)\end{array}$ & $\begin{array}{l}0,3(0,1+0,2) \\
2,5(0,8+1,7) \\
1,7(0,5+1,2)\end{array}$ \\
\hline & $3 \%$ & $\begin{array}{l}15 \\
15 \\
30\end{array}$ & $\begin{array}{l}0,9(0,2+0,7) \\
1,3(0,2+1,1) \\
4,5(2,4+2,1)\end{array}$ & $\begin{array}{l}1,1(0,55+0,55) \\
1,2(0,1+1,1) \\
3,0(1,8+1,2)\end{array}$ & $\begin{array}{l}0,4(0,15+0,25) \\
0,6(0,2+0,4) \\
2,7(1,0+1,7)\end{array}$ & $\begin{array}{l}0,2(0,02+0,2) \\
0,5(0,1+0,4) \\
1,7(0,6+1,1)\end{array}$ \\
\hline
\end{tabular}
the cleaning and disinfection efficiencies, are presented.

$1 \%: 1 \%(\mathrm{~V} / \mathrm{v})$ de solution de nettoyage-désinfection ; $3 \%: 3 \%(\mathrm{~V} / \mathrm{V})$ de solution de nettoyage-désinfection. $\mathrm{R}_{0}:$ rugosité moyenne $0,25 \mu \mathrm{m} ; R_{1}$ : rugosité moyenne $0,73 \mu \mathrm{m} . Q_{0}: 0,01 \mathrm{~g}$ de souillure par éprouvette $; Q_{1}: 0,1 \mathrm{~g}$ de souillure par éprouvette.

$1 \%: 1 \%(\mathrm{~V} / \mathrm{V})$ of detergent-disinfectant solution; $3 \%=3 \%(\mathrm{~V} / \mathrm{V})$ of detergent-disinfectant solution; $R_{0}: 0.25 \mu \mathrm{m}$ average roughness ; $R_{1}: 0.73 \mu \mathrm{m}$ average roughness; $Q_{0}: 0.01 \mathrm{~g}$ soil per sample; $Q_{1}: 0.1 \mathrm{~g}$ soil per sample. 
souillure témoin, mesurée par la proportion de spores dénombrées après insonification et broyage péristaltique, peut être considérée comme totale: $98 \%$ dans le cas du caillé, $107 \%$ dans le cas du saintpaulin. Dans les 2 cas, le coefficient de variation est de $11 \%$.

Dans les souillures, le dénombrement du marqueur de désinfection est compris entre $10^{5}$ et $10^{6}$ UFC/éprouvette avec la quantité de souillure $Q_{0}$, et entre $10^{7}$ et $10^{8}$ UFC/éprouvette avec la quantité $Q_{1}$.

\section{Efficacité du procédé}

Les efficacités mesurées se révèlent faibles en général : l'efficacité globale n'atteint 4,5 que pour une souillure (le saintpaulin) (tableau I). C'est d'ailleurs dans ce seul cas que l'éprouvette a un aspect propre à l'œil nu, mais une efficacité de 4,5 sur une souillure contenant $10^{7}$ UFC signifie qu'il reste encore $3.10^{2}$ UFC.

Avec les 2 souillures, le nettoyage apporte à l'efficacité globale une contribution un peu plus grande que la désinfection.

\section{Influence des facteurs expérimentaux}

La concentration de désinfectant $\mathrm{C}$ ne participe pas de façon significative à l'explication de la variance (tableau II). En revanche, les autres facteurs interviennent, notamment le temps $T$ et la quantité de souillure $Q$, et à un degré moindre, la nature de la souillure $S$. La rugosité $R$ intervient peu, et seulement sur le nettoyage. Les interactions significatives participent peu à l'explication de la variance, sauf l'interaction entre le temps $T$ et la nature de la souillure $S$ : cette interaction a même la plus importante contribution à l'explication de la variance.

\section{DISCUSSION}

Le procédé de nettoyage-désinfection qui vient d'être évalué n'est utilisé que pour apprécier la méthode de mesure de l'efficacité du nettoyage et de la désinfection que nous avons mise au point, et pour vérifier si cette méthode répond au cahier des charges que nous nous sommes fixé. Ce n'est donc qu'après avoir discuté cette méthode que nous reviendrons sur le procédé de nettoyage-désinfection utilisé ici.

\section{Évaluation de la méthode mise au point}

Le premier point du cahier des charges concerne les souillures. Nous allons discuter 3 aspects de celles-ci.

\section{La quantité}

Les résultats indiquent une efficacité limitée du procédé. Or, celui-ci doit normalement être accompagné d'un brossage. Nous avons pu vérifier au cours d'un autre plan d'expérience destiné à comparer 2 produits détergents-désinfectants combinés, qu'en effet, le rôle du brossage venait en second sur la liste des facteurs d'explication de la variance, après la nature du produit (Brouillaud-Delattre, 1993). L'appréciation quantitative du nombre de bactéries n'est pas affectée par le fait que toutes les manipulations ne sont pas aseptiques. En effet, les recontaminations sont peu nombreuses en comparaison avec l'effectif des populations bactériennes étudiées et les milieux de culture sont spécifiques; en outre, dans le cas du dénombrement des spores, la probabilité de recontamination par des bactéries thermophiles est quasi nulle. 
Tableau II. Pourcentages d'explication de la variance, et signification des facteurs et de leurs interactions.

Percentage of variance accounted for significance of factors and factor interactions. Only significant factors and interactions are shown.

\begin{tabular}{|c|c|c|c|}
\hline Facteurs & Nettoyage & Désinfection & Nettoyage + désinfection \\
\hline $\begin{array}{l}\mathrm{T} \\
\mathrm{C} \\
\mathrm{CT}\end{array}$ & $24,6^{\star \star *}$ & $12,9^{* * *}$ & $22,0^{* * *}$ \\
\hline S & $14,4^{* * *}$ & $4,9 * * *$ & $10,9^{* * *}$ \\
\hline$Q$ & $8,8^{* * *}$ & $15,9^{\star \star *}$ & $14,4^{* * *}$ \\
\hline$\vec{R}$ & $2,2^{*}$ & & $1,5^{\star}$ \\
\hline $\begin{array}{l}\text { QS } \\
\text { RS }\end{array}$ & $1,6^{*}$ & & \\
\hline $\mathrm{CS}$ & & & \\
\hline $\begin{array}{l}\text { ST } \\
\text { QR }\end{array}$ & $20,7^{\star \star \star}$ & $35,7^{\star \star \star}$ & $33,0^{\star \star \star}$ \\
\hline $\mathrm{CQ}$ & & & \\
\hline $\begin{array}{l}\text { QT } \\
\text { CR }\end{array}$ & $2,7^{*}$ & $5,2^{* *}$ & \\
\hline RT & & & \\
\hline QRS & & & \\
\hline CQS & $4,5^{\star *}$ & & $2,4^{*}$ \\
\hline QST & & $2,3^{*}$ & \\
\hline CRS & $1,6^{\star}$ & & $1,7^{\star}$ \\
\hline RST & $2,4^{*}$ & & $1,4^{*}$ \\
\hline CST & $3,0^{* *}$ & & \\
\hline CQR & & & \\
\hline CQT' & & $1,9^{*}$ & \\
\hline CRT & & $3,1^{*}$ & \\
\hline
\end{tabular}

$T$ : temps d'application du procédé ; $\mathrm{C}$ : concentration du produit nettoyant-désinfectant ; $\mathrm{S}$ : nature de la souillure ; $\mathrm{Q}$ : quantité de souillure $\mathrm{R}$ : rugosité de la surface. $\because, \cdots, *$ : significatif respectivement au seuil $0,05,0,01 ; 0,001$.

$T$ : time of process application; $C$ : concentration of detergent-disinfectant; $S$ : soiling material; $Q$ : soil amount; $R$; surface roughness. ; $: \cdots: \mathrm{P} \leq 0.05,0.01,0.001$.

\section{La nature}

Les souillures utilisées ici sont représentatives, par leur composition, de souillures de l'industrie laitière. Toutefois, nous avons dû y ajouter une proportion non négligeable de liquide de suspension des marqueurs, car il n'a pas été possible de mélanger ceux-ci sous forme de culot de centrifugation: ils restaient sous forme d'amas, rendant impossible la poursuite de l'expérimentation. Le citrate a alors été choisi en raison de ses propriétés de dispersion des produits laitiers bien connues (Tsai et Cousin, 1990).

\section{La formation de biofilm}

La croissance des bactéries a été faible dans le saint-paulin, mais forte dans le 
caillé. Les photographies au microscope électronique à balayage de biofilm obtenues avec le même mélange de souches bactériennes montrent l'aspect d'un biofilm épais formé en une nuit d'incubation sur du lait (Brouillaud-Delattre et Cerf, 1993).

Le second point du cahier des charges concerne la reproductibilité des essais. La variabilité entre blocs, mesurée grâce à la répétition de 2 blocs, est du même ordre de grandeur que la variabilité à l'intérieur d'un bloc. Cela signifie qu'il n'y a ni variation entre blocs, ni dérive de l'erreur systématique, et que l'on peut comparer les 6 blocs comme s'ils ne constituaient qu'un seul et même essai. L'objectif de reproductibilité est donc atteint.

Le troisième point du cahier des charges est la caractérisation des procédés de nettoyage et de désinfection. La méthode permet effectivement de quantifier séparément l'efficacité du nettoyage et de la désinfection. Toutefois, la quantification est d'autant meilleure que l'efficacité globale des procédés est grande (Brouillaud-Delattre, 1993).

Le quatrième point du cahier des charges est de permettre l'emploi de plans d'expérience multifactoriels. Cela a été obtenu en faisant de sorte que 16 éprouvettes puissent être soumises simultanément au procédé essayé.

\section{Facteurs influant sur l'efficacité du procédé essayé}

Bien que le procédé ne soit pas l'objet essentiel de l'étude, nous commenterons brièvement les résultats relatifs à l'efficacité du procédé. Examinons d'abord l'influence du temps et de la quantité de souillure : ces facteurs agissent de façon prévisible, l'augmentation du temps se tra- duisant par une meilleure efficacité du nettoyage et de la désinfection ; la quantité de souillure agit en sens inverse.

La nature de la souillure est importante : le caillé est moins bien nettoyé et désinfecté que le saint-paulin. Cette observation est compatible avec le fait que le caillé est plus compact, et l'observation industrielle indique qu'il colle davantage aux surfaces.

La nature de la souillure et le temps d'application du produit sont à l'origine d'une interaction importante et très hautement significative. Cela signifie que la cinétique de nettoyage-désinfection par le produit étudié est liée de façon très hautement significative à la nature de la souillure.

Le plan d'expérience ne montre pas de différence significative d'efficacité entre les 2 concentrations étudiées. En effet, l'efficacité de la désinfection est faible dans tous les cas : $\leq 1,3$ avec le caillé, $\leq 2,4$ avec le saint-paulin. Dans ces conditions, on peut faire l'hypothèse que la molécule active, qui contient du chlore, est rapidement inactivée, quelle que soit sa concentration, par les matières organiques des souillures. En outre, les caractéristiques physiques des souillures s'opposent probablement à la diffusion de la molécule désinfectante en leur sein. Cette hypothèse est rendue vraisemblable par le fait que cette même molécule est très efficace lorsque la souillure est constituée par du lait (BrouillaudDelattre, 1993). Enfin, il doit aussi y avoir un effet de l'état physiologique des bactéries dans le biofilm : en effet, une comparaison de la résistance au désinfectant des bactéries cultivées soit en suspension dans le lait, soit en culture sur une surface recouverte de lait, montre que dans le second cas, la résistance est plus élevée (Brouillaud-Delattre, 1993). Cette expérience ne peut être répétée avec une 
souillure à base de fromage, qui n'est pas liquide.

L'influence de la rugosité est très faible. On peut penser que les 2 valeurs choisies pour les essais ne sont pas suffisamment différentes l'une de l'autre, et qu'en outre l'effet de la rugosité a été masqué par les grandes quantités de souillures déposées et leur texture.

Le produit testé a été peu efficace: $E_{g}$ est resté inférieur à 3,5 , sauf dans un cas où il a atteint 4,5 (tableau I). Cela s'explique vraisemblablement par la quantité excessive de souillure utilisée. Un tel résultat confirme que seules des surfaces peu souillées ou bien nettoyées peuvent être bien désinfectées ; en aucun cas, les résultats obtenus présentés ici ne peuvent être extrapolés à d'autres produits, souillures, types de surface, etc.

\section{CONCLUSION}

La méthode mise au point (souillure représentative transformée en biofilm; étalée, nettoyée et désinfectée avec un robot; comportant des marqueurs spécifiques pour la mesure séparée du nettoyage et de la désinfection; et utilisant des plans d'expérience multifactoriels) apporte un moyen de mesurer l'efficacité des procédés de nettoyage et de désinfection pour les surfaces ouvertes. C'est la première fois à notre connaissance qu'une méthode devient disponible pour mesurer le nettoyage, et c'est aussi la première fois qu'un biofilm contenant 2 espèces bactériennes est utilisé. Cette méthode s'applique à toutes les industries alimentaires à condition de choisir des souillures et des microorganismes représentatifs des conditions industrielles. Elle permet à la fois de comparer des procédés entre eux, et d'optimi- ser les conditions d'application d'un procédé donné.

\section{REMERCIEMENTS}

Les auteurs remercient A Sadoudi pour son aide lors de la fabrication du robot. Ce travail a été réalisé grâce au concours financier du ministère de la Recherche et de la Technologie et de I'Association pour le développement de la recherche dans l'industrie laitière.

\section{RÉFÉRENCES}

AFNOR (1989) Détermination de l'activité sporicide. Antiseptiques et désinfectants utilisés à l'état liquide, miscibles à l'eau et neutralisables. Norme française NF T 72-230 (1989), AFNOR, Paris-La Défense

Brouillaud-Delattre A (1993) Mise au point d'un robot prototype permettant de tester les produits de nettoyage et de désinfection utilisés dans les industries laitières. Thèse Doctorat, Univ Montpellier II

Brouillaud-Delattre A, Cerf O (1993) Le biofilm: une réalité industrielle. Rev Lait Fr 528, 3031

Cerf O, Carpentier B (1992) Un point critique pour la maîtrise de l'hygiène: l'environnement des ateliers. In: Microbiologie prédictive et HACCP (Amgar A, éd). ASEPT, Laval, $171-180$

Characklis WG, Marshall KG (1990) Biofilms : a basis for an interdisciplinary approach. In : Biofilms (Characklis WG, Marshall KG, eds) John Wiley and Son, New York, 3-15

Costerton JW, Geesey GG, Cheng KG (1978) Comment collent les bactéries. Pour Sci 5 , 100-110

De Man JC, Rogosa M, Sharpe ME (1960) A medium for the cultivation of lactobacilli. $J$ Appl Bacteriol 23, 130-135

Elliker PR, Anderson AW, Hannesson G (1956) An agar culture medium for lactic acid strep- 
tococci and lactobacilli. J Dairy Sci 39, 16111612

Galesloot TE, Radema L, Kooy EG, Hup G (1967) A sensitive method for the evaluation of cleaning processes with a special version adapted to the study of the cleaning of tanks. Neth Milk Dairy J21, 214-222

Goupy J (1988) La méthode des plans d'expérience. Dunod, Paris
Holah JT, Venema-Keur BM, Trägårdh C, Illi $\mathrm{H}$, Lalande M, Cerf O (1992) A method for the assessment of in-place cleanability of food processing equipment. Trends Food Sci Technol 3, 325-328

Tsai GJ, Cousin MA (1990) Enzyme-linked immunoabsorbent assay for detection of molds in cheese and yogurt. J Dairy Sci 73, 33663378 